\title{
Analytical challenges and advancements in bioanalysis of therapeutic proteins
}

\author{
Snehal K Shukla ${ }^{1}$ \& Vivek Gupta*,1 \\ ${ }^{1}$ College of Pharmacy \& Health Sciences, 8000 Utopia Parkway, St John's University, Queens, NY 11439, USA \\ *Author for correspondence: Tel.: +1 718990 3929; guptav@stjohns.edu
}

First draft submitted: 8 January 2020; Accepted for publication: 15 January 2020; Published online: 21 February 2020

Keywords: analytical • biosimilars • capillary electrophoresis • challenges • liquid chromatography • therapeutic proteins

\section{Therapeutic proteins \& associated challenges}

Therapeutic proteins have been widely explored for the treatment of several diseases resulting into development of novel therapies. The protein therapeutics class comprises peptides, recombinant proteins, monoclonal antibodies, antibody-drug conjugates, antibody fusion proteins and antibody fragments [1]. In the past two decades, therapeutic proteins have gained significant success with clinical significance resulting into several US FDA approved therapies with improved patient care. Therapeutic proteins encounter critical challenges during their production and designing of formulations. The challenges can be attributed to the complexity of therapeutic proteins with respect to high molecular weight, inherent heterogeneity, requirement for post-translation modifications and production from broad range of organisms leading to the presence of a variety of biological matrices during production [2]. These macromolecules are prone to be affected by microheterogeneities resulting either from their intrinsic complex nature or chemical/enzymatic modifications subjected during their production or storage conditions, or interactions with host cell proteins [3]. While the developed microheterogeneities may demonstrate mild-to-moderate impact in most cases, they may also trigger structural or conformational changes in other cases resulting into severe consequences raising concerns about the safety of developed therapeutic protein [4].

\section{Importance of characterization \& analytical tools for macromolecular therapies}

The production and manufacturing of therapeutic proteins is a complex and challenging process involving several critical intermediate steps. Therapeutic efficacy and adverse effects of these proteins are significantly affected by their structure and composition and in certain cases are modulated due to conformational changes [5]. Therefore, the exact structure and composition of therapeutic proteins are crucial as minor changes may lead to unwanted side effects and possible loss of therapeutic activity. Also, the detailed structural analysis of therapeutic proteins would help to facilitate development of successful biosimilars [6]. Owing to such vital requirements, it is essential to characterize the therapeutic proteins at every step of production and processing along with final end products.

\section{Analytical approaches serving as bioanalytical tools}

Remarkable advancements in analytical field over the last few decades have led to development of various technologies efficient in elucidating complex structures. The protein characterization techniques have evolved dramatically; from x-ray crystallography being used to determine the first protein structure to the widely used NMR [4]. However, these analytical methods are known to have limited abilities such as $\mathrm{x}$-ray crystallography can only determine the conformation of the unfolded protein structures, while NMR involves molecular weight limitations and incompetency in determination of amino acid composition [4]. Other commonly used analytical techniques for protein characterization are size-exclusion (SE) chromatography and analytical ultracentrifugation; for determination of protein aggregates and fragmentation, circular dichroism and differential scanning calorimetry; for determination of protein folding, stability and interaction and microflow digital imaging for visualization of any visible particles [5]. These techniques provide limited information about the protein while the detailed constitutional information remains unrevealed. 
Numerous chromatographic approaches such as SE, affinity chromatography, hydrophilic/hydrophilic interaction and reverse phase have been indispensably used for characterization of therapeutic proteins [6]. Several modes of LC have been employed for assessing physicochemical characterization of therapeutic proteins, quantitative analysis in various biological matrices, determining pharmacokinetic profile of therapeutic proteins and determination of post-translation modifications [7]. MS has established success for characterization of macromolecules and has been widely exploited to analyze protein structures and determine molecular weight. Native MS is used for qualitative and quantitative determination of intact protein under non-denaturing environment. This approach is however known to suffer due to intensive manual work hampering its implementation to the automated advancements [8].

Electrophoresis is another routinely used technique for separation of complex therapeutic proteins with respect to their size along with assessment of impurities. Traditional SDS-PAGE is widely employed for separating contaminants and monitoring presence of protein aggregates. However, it is limited to only qualitative analysis and is also known to be have issues of non-reproducibility [9]. CE has gained significance as a distinct electrophoretic technique for determination of heterogeneity based on size and charge along with adequate monitoring of post-translational modifications [10]. CE such as capillary gel, capillary isoelectric focusing and capillary zone electrophoresis are known to possess advantages such as faster rate of separation, reduced run time, requirement of lower sample volume and enhanced resolution [11]. While efficacious, CE is known to be affected by adsorption of analytes which in turn impacts the sensitivity and reproducibility of the method, thereby limiting its clinical applications.

\section{Implication of hyphenation techniques}

Hyphenation approach involves coupling of a separation technique with a detection methodology for exploiting the advantages of both the methods and overcoming the existing limitations. MS is the most used detection technique coupled with chromatographic or electrophoretic separation for characterization of therapeutic proteins. LC-MS has been extensively used for determining mass distribution, presence of complexes and conformation of therapeutic proteins [12]. Furthermore, LC-MS/MS is exploited for assessing the presence of protein (top-down approach) and protein species (bottom-up approach), determination of partial sequences and molecular weight of intact species, identification of protein species and post-translational modifications [13]. These hyphenation methods are also useful in determining the secondary and tertiary structures of proteins along with composition of the protein species and identification of post-translation modifications [12]. However, these methods also face several challenges including intensive sample preparation prior to analysis, requirement of spectral libraries to determine the structure and composition of analyzed therapeutic protein and in some cases may require high amount of sample and provide limited information about the protein species [14]. Another hyphenation approach, SE-LC is employed for determining the purity, analyzing heterogeneity of charges and some specific post-translational modifications [15]. SE-LC can be further coupled with MS as an indirect interface since direct coupling is limited due to presence of high amount of salts, poor peak shape and impact on protein conformation [8].

The most recently used combinatorial approach of CE-MS has proven to be an indisputable strategy for characterization of therapeutic proteins resulting in development of fast, sensitive and specific techniques [16]. This strategy helps in determination of molecular weight of intact proteins, determining the amino acid composition as well as any post-translational modifications [17]. Consequently, to improve and miniaturize the electrophoresis technique, micro-chip electrophoresis has also been developed [18]. This technique has significantly reduced the time for analysis in addition to reduction in sample volume with increased efficiency of resolution, therefore making it a cost-effective technique $[18,19]$.

\section{Conclusion}

The development of therapeutic proteins is a highly complex process wherein several critical attributes are required to be continuously monitored. Advances in the analytical field have improved the protein characterization significantly. However, each analytical method has its own limitations that impact the protein characterization in its own unique way. Existing limitations of analytical methodologies are being resolved using combinatorial/hyphenation approaches; wherein two technologies are coupled to overcome the limitations and impart synergistic effect for effective characterization of therapeutic proteins. These emerging hyphenation technologies impart clinical significance to the developed bioanalytical methods, help in development of biosimilars, provide high-throughput production setting, and improve the quality of therapeutic products. 


\section{Financial \& competing interests disclosure}

This work was supported by an NIH Research Enhancement Award (R15), 1R15HL138606-01A1 to V Gupta and SK Shukla was supported by research assistantship from NIH-R15 to V Gupta. The authors have no other relevant affiliations or financial involvement with any organization or entity with a financial interest in or financial conflict with the subject matter or materials discussed in the manuscript apart from those disclosed.

No writing assistance was utilized in the production of this manuscript.

\section{References}

1. Schluter H, Fuh M, Steffen P. Tools for the analysis and characterization of therapeutic protein species. BS 2016(6), 17 (2016).

2. Bandaranayake AD, Almo SC. Recent advances in mammalian protein production. FEBS Lett. 588(2), 253-260 (2014).

3. Gahoual R, Heidenreich A-K, Somsen GW et al. Detailed characterization of monoclonal antibody receptor interaction using affinity liquid chromatography hyphenated to native mass spectrometry. Anal. Chem. 89(10), 5404-5412 (2017).

4. Kaltashov IA, Bobst CE, Abzalimov RR, Wang G, Baykal B, Wang S. Advances and challenges in analytical characterization of biotechnology products: mass spectrometry-based approaches to study properties and behavior of protein therapeutics. Biotechnol. Adv. 30(1), 210-222 (2012).

5. Federici M, Lubiniecki A, Manikwar P, Volkin DB. Analytical lessons learned from selected therapeutic protein drug comparability studies. Biologicals 41(3), 131-147 (2013).

6. Tsiftsoglou AS, Ruiz S, Schneider CK. Development and regulation of biosimilars: current status and future challenges. BioDrugs 27(3), 203-211 (2013).

7. Fekete S, Guillarme D, Sandra P, Sandra K. Chromatographic, electrophoretic, and mass spectrometric methods for the analytical characterization of protein biopharmaceuticals. Anal. Chem. 88(1), 480-507 (2016).

8. Ehkirch A, Hernandez-Alba O, Colas O, Beck A, Guillarme D, Cianférani S. Hyphenation of size exclusion chromatography to native ion mobility mass spectrometry for the analytical characterization of therapeutic antibodies and related products. J. Chromatogr. B Analyt. Technol. Biomed. Life Sci. 1086, 176-183 (2018).

9. Zhu Z, Lu JJ, Liu S. Protein separation by capillary gel electrophoresis: a review. Anal. Chim. Acta 709, 21-31 (2012).

10. Han M, Rock BM, Pearson JT, Rock DA. Intact mass analysis of monoclonal antibodies by capillary electrophoresis - mass spectrometry. J. Chromatogr. B 1011, 24-32 (2016).

11. Zhao SS, Chen DDY. Applications of capillary electrophoresis in characterizing recombinant protein therapeutics. Electrophoresis 35(1), 96-108 (2014).

12. Angel TE, Aryal UK, Hengel SM et al. Mass spectrometry-based proteomics: existing capabilities and future directions. Chem. Soc. Rev. 41(10), 3912 (2012).

13. Zhang H, Cui W, Gross ML. Mass spectrometry for the biophysical characterization of therapeutic monoclonal antibodies. FEBS Lett. 588(2), 308-317 (2014).

14. Lanucara F, Holman SW, Gray CJ, Eyers CE. The power of ion mobility-mass spectrometry for structural characterization and the study of conformational dynamics. Nat. Chem. 6(4), 281-294 (2014).

15. Brower KP, Ryakala VK, Bird R et al. Single-step affinity purification of enzyme biotherapeutics: a platform methodology for accelerated process development. Biotechnol. Prog. 30(3), 708-717 (2014).

16. Mikšík I. Coupling of CE-MS for protein and peptide analysis. J. Sep. Sci. 42(1), 385-397 (2019).

17. Faserl K, Sarg B, Gruber P, Lindner HH. Investigating capillary electrophoresis-mass spectrometry for the analysis of common post-translational modifications. Electrophoresis 39(9-10), 1208-1215 (2018).

18. Štěpánová S, Kašička V. Analysis of proteins and peptides by electromigration methods in microchips. J. Sep. Sci. 40(1), 228-250 (2017).

19. Dawod M, Arvin NE, Kennedy TR. Recent advances in protein analysis by capillary and microchip electrophoresis. Analyst 142(11), 1847-1866 (2017). 